The University of Maine

DigitalCommons@UMaine

Marine Sciences Faculty Scholarship

School of Marine Sciences

8-1-2013

\title{
Influence of Raman scattering on ocean color inversion models
}

Toby K. Westberry

Oregon State University

Emmanuel Boss

University of Maine, emmanuel.boss@maine.edu

Zhongping Lee

University of Massachusetts Boston

Follow this and additional works at: https://digitalcommons.library.umaine.edu/sms_facpub

Part of the Oceanography and Atmospheric Sciences and Meteorology Commons

\section{Repository Citation}

Westberry, Toby K.; Boss, Emmanuel; and Lee, Zhongping, "Influence of Raman scattering on ocean color inversion models" (2013). Marine Sciences Faculty Scholarship. 163.

https://digitalcommons.library.umaine.edu/sms_facpub/163

This Article is brought to you for free and open access by DigitalCommons@UMaine. It has been accepted for inclusion in Marine Sciences Faculty Scholarship by an authorized administrator of DigitalCommons@UMaine. For more information, please contact um.library.technical.services@maine.edu. 


\title{
Influence of Raman scattering on ocean color inversion models
}

\author{
Toby K. Westberry, ${ }^{1, \star}$ Emmanuel Boss, ${ }^{2}$ and Zhongping Lee ${ }^{3}$ \\ 'Department of Botany and Plant Pathology, Oregon State University, Corvallis, Oregon 97331-2902, USA \\ ${ }^{2}$ School of Marine Sciences, University of Maine, Orono, Maine 04469-5706, USA \\ ${ }^{3}$ Environmental, Earth, and Ocean Sciences Department, University of Massachusetts Boston, Boston, \\ Massachusetts 02125, USA \\ ${ }^{*}$ Corresponding author: toby.westberry@science.oregonstate.edu
}

Received 18 March 2013; revised 12 June 2013; accepted 6 July 2013;

posted 8 July 2013 (Doc. ID 187324); published 1 August 2013

\begin{abstract}
Raman scattering can be a significant contributor to the emergent radiance spectrum from the surface ocean. Here, we present an analytical approach to directly estimate the Raman contribution to remote sensing reflectance, and evaluate its effects on optical properties estimated from two common semi analytical inversion models. For application of the method to ocean color remote sensing, spectral irradiance products in the ultraviolet from the OMI instrument are merged with MODerate-resolution Imaging Spectroradiometer (MODIS) data in the visible. The resulting global fields of Raman-corrected optical properties show significant differences from standard retrievals, particularly for the particulate backscattering coefficient, $b_{b p}$, where average errors in clear ocean waters are $\sim 50 \%$. Given the interest in transforming $b_{b p}$ into biogeochemical quantities, Raman scattering must be accounted for in semi analytical inversion schemes. (C) 2013 Optical Society of America

OCIS codes: (010.4450) Oceanic optics; (010.0280) Remote sensing and sensors; (010.5630) Radiometry; (010.1690) Color.

http://dx.doi.org/10.1364/AO.52.005552
\end{abstract}

\section{Introduction}

Ocean color inversion models provide a means of relating the emergent radiance spectrum to various absorbing and scattering components in the surface ocean [1]. In turn, these absorption and scattering indices convey rich information on suspended and dissolved materials that can now be estimated from satellite at global, synoptic scales. Some of the better characterized bio-optical signals (e.g., chlorophyll or water transparency) have been used to examine longterm changes in ocean properties associated with climate variability [2-4]. However, the accuracy of retrieved quantities depends upon a number of factors ranging from satellite sensor calibration to

$1559-128 X / 13 / 225552-10 \$ 15.00 / 0$

(C) 2013 Optical Society of America the formulation of the inversion algorithm itself. The latter depends upon the ability to account for all significant processes affecting light transmission and propagation in the ocean and atmosphere. Some of these processes and relationships can be expressed analytically in an inversion algorithm, while others rely on empirically derived information.

One such physical process that affects the ambient light field is Raman scattering. Raman scattering is a quantum molecular phenomenon that results from photon interactions with the medium itself (e.g., seawater), and which are re-emitted at wavelengths differing from the excitation source [5,6]. While Raman scattering is often conceptually lumped together with other "trans-spectral" processes, it is a fundamentally different process from other forms of absorption-reemission interactions, such as fluorescence. Unlike fluorescence, Raman scatter is 
associated with a fixed spectral shift between excitation and emission frequencies [6]. In order to estimate the spectral intensity of Raman scattered light, three pieces of information must be known: the shape and magnitude of the excitation energy spectrum, how that excitation energy is spectrally redistributed, and some metric quantifying the "efficiency" of Raman scatter. The first two pieces are simply the incident irradiance spectrum and the spectral redistribution function, needed to predict the emission spectra [7]. Therefore, most of the effort in ocean optics related to Raman scatter has centered on characterizing the third piece, the Raman scattering cross section [8-11]. Gordon [12] provides a brief chronology of these measurements and how they compare with various simulation [13-15] and fieldbased studies $[16,17]$.

The results of these past efforts demonstrate that Raman scattering can contribute significantly to the marine upwelling radiance field across all visible wavelengths to a variable degree $[8,14,17,18]$. For example, Waters [15] carried out Monte Carlo simulations suggesting that the Raman fraction of water leaving irradiance could be as high as $\sim 15 \%$, but this was limited to longer wavelengths $(\lambda>650 \mathrm{~nm})$. Further, the effect decreased rapidly with increasing pigment biomass ( $\sim 2 \%$ when chlorophyll concentration $\left.(C h l)=1.0 \mathrm{mg} \mathrm{m}^{-3}\right)$. More recently, however, Gordon [12] modeled the Raman effect on the upwelling (ir) radiance and found it to be higher than previous studies ( $>25 \%$ of $L_{u}(\lambda)$, spectral upwelling radiance, in pure seawater for $\lambda>500 \mathrm{~nm}$ ) and significant for chlorophyll concentrations up to $5 \mathrm{mg} \mathrm{m}^{-3}$. Both of these results-a higher relative contribution from Raman and a decreased dependence on $\mathrm{Chl}$-were attributed to the use of revised pure water absorption values [19] and newly reported spectral variability of the Raman scattering cross section [10]. Gordon concluded that Raman scattering “...cannot be ignored in ocean color modeling." In subsequent work, Gordon et al. [20] also demonstrated that Raman scatter prevented optical closure efforts using hyperspectral radiometric field measurements. Thus, it should be evident that Raman scatter can introduce uncertainty to models relating the radiance field to the inherent optical properties (IOPs) of the ocean.

Despite the presumed importance of Raman scattering, few studies have included it in semi analytical algorithms designed to invert in situ or satellite ocean color reflectance data. In particular, three such algorithms have been put forward [21-23], but their implementation of Raman scatter has not been carried forward in subsequent studies or in the comprehensive report by the IOCCG [24]. This may be due, in part, to the fact that ocean color remote sensing satellites do not currently have ultraviolet (UV) bands, which are needed to compute Raman excitation in the blue part of the visible spectrum. Indeed, these efforts have relied on empirical relationships or lookup tables derived from radiative transfer simulations. Here, we present a simulated dataset to examine the effects of Raman scattering on IOP inversion products from semianalytical models. We then develop an approach to directly estimate the inelastic Raman contribution to remote sensing reflectance and the resulting effects of its removal on inversion of simulated and satellite ocean color data.

\section{Methods}

\section{A. Radiative Transfer Simulations}

A series of radiative transfer simulations (HydroLight, Sequoia Scientific, Inc.) were generated to (1) demonstrate the magnitude of the Raman effect on $R_{\mathrm{rs}}(\lambda)$ (remote-sensing reflectance, the ratio of water-leaving radiance to downwelling irradiance just above the surface), and (2) provide a validation dataset for an approach to remove the Raman contribution to $R_{\mathrm{rs}}(\lambda)$. Paired runs with and without Raman scatter were simulated for Chl ranging from 0.01 to $5.0 \mathrm{mg} \mathrm{m}^{-3}(C h l=0.01,0.02,0.03,0.04$, $\left.0.07,0.1,0.2,0.3,0.5,0.7,1.0,2.0,5.0 \mathrm{mg} \mathrm{m}^{-3}\right)$. In order to efficiently incorporate Raman scattering into the radiative transfer equation, HydroLight uses an azimuthally averaged formulation of Raman scatter, which gives the correct Raman contribution to irradiances and nadir radiances (see Appendix A in [25]). The standard "Case 1" model embedded in HydroLight was used to relate $C h l$ to other IOPs, details of which can be found in Gordon and Morel [26], Morel and Maritorena [27]. A fixed particle phase function (Fournier-Forand) was used with a particulate backscattering ratio $\left(b_{b p} / b_{p}\right)$ equal to 0.01 . In this model, pure seawater properties are specified by Pope and Fry [19] and Smith and Baker [28] for absorption and scattering, respectively. For all simulations, a clear sky with solar zenith angle of $30^{\circ}$ and a wind speed of $5 \mathrm{~m} \mathrm{~s}^{-1}$ was assumed.

\section{B. Satellite Remote Sensing Data}

Satellite products from two independent sensors were used in this work, the ozone mapping instrument (OMI) on Aura and the MODerate-resolution Imaging Spectroradiometer (MODIS) on Aqua. Both Aura and Aqua are part of the A-train constellation of Earth observing satellites, with Aura having an Equatorial crossing time just a few minutes later than Aqua ( 1:30 pm). OMI data were obtained as daily, Level 3 products from Goddard Earth Sciences Data and Information Sciences Center (GES DISC), and were temporally binned to create monthly composites. These data consist of noon-time UV irradiances at four fixed wavelengths $(305,310,324$ and $380 \mathrm{~nm}$ ). From MODIS, monthly Level 3 products of instantaneous PAR $(i P A R)$ and spectral satellite remote sensing reflectances, $R_{\mathrm{rs}}(\lambda)$, were downloaded directly from the NASA Ocean Color Web portal (http://oceancolor.gsfc.nasa.gov/). $i P A R$ was decomposed to estimate spectral downwelling irradiance $\left(E_{d}(\lambda)\right)$ using fixed fractions estimated from an 
atmospheric radiative transfer model [29]. The fractional constants are stable within $1 \%-6 \%$ depending on wavelength and illumination conditions. Together, resultant $E_{d}(\lambda)$ in the visible and OMI UV flux data allow pixel-wise reconstruction of incident irradiance spectra. $E_{d}(\lambda)$ at Raman excitation wavelengths corresponding to MODIS visible bands (365, $387.5,421,452.5,467.5 \mathrm{~nm}$ for MODIS bands 8-12) were linearly interpolated and band-averaged (bandwidths $\sim 10 \mathrm{~nm})$. Global fields of MODIS $R_{\mathrm{rs}}(\lambda)$ were used to estimate IOPs using two semianalytical inversion models; the Garver-Siegel-Maritorena (GSM) model [30] and the quasi-analytical algorithm (QAA) [31]. Retrieved IOPs include the phytoplankton absorption $\left(a_{p h}(\lambda)\right.$, QAA only) or chlorophyll concentration ( $C h l$, GSM only), particulate backscattering coefficients $\left(b_{b p}(\lambda)\right)$, and dissolved and detrital organic matter absorption coefficient $\left(a_{\mathrm{CDM}}(\lambda)\right)$.

C. Calculation of Raman Contribution to Remote Sensing Reflectance

We build on the approaches of Bartlett [18], Sathyendranath and Platt [22], and Loisel and Stramski [23] and express the remote sensing reflectance $\left(R_{\mathrm{rs}}\right)$ as

$$
R_{\mathrm{rs}}\left(\lambda, 0^{-}\right)=R_{\mathrm{rs}, E}\left(\lambda, 0^{-}\right)+R_{\mathrm{rs}, I E}\left(\lambda, 0^{-}\right),
$$

where the first term on the right-hand side accounts for the contribution to $R_{\mathrm{rs}}$ from elastic scattering (subscript $E$ ), and the second term accounts for inelastic scattering (subscript $I E$ ). For this exercise, we are functionally considering three distinct processes as sources of "inelastic scattering" that affect remotely sensed ocean color; fluorescence from colored dissolved material (CDOM) and chlorophyll, and Raman scatter from seawater itself. However, CDOM fluorescence can be considered negligible [32], and chlorophyll fluorescence only impacts $\overline{R_{\mathrm{rs}}}$ near $685 \mathrm{~nm}$ [33]. Hence, we assume that at all wavelengths except near $685 \mathrm{~nm}$ :

$$
R_{\mathrm{rs}}\left(\lambda, 0^{-}\right)=R_{\mathrm{rs}, E}\left(\lambda, 0^{-}\right)+R_{\mathrm{rs}, \operatorname{Raman}}\left(\lambda, 0^{-}\right) .
$$

The immediate goal of semianalytical inversion algorithms is to obtain the spectral absorption and backscattering coefficients for given $R_{\mathrm{rs}, E}\left(\lambda, 0^{-}\right)$ of the surface ocean. Therefore, we must first estimate $R_{\mathrm{rs}, \operatorname{Raman}}\left(\lambda, 0^{-}\right)$and remove it from measured $R_{\mathrm{rs}}\left(\lambda, 0^{-}\right)$. The Raman scattering coefficient, $b^{R}\left(\lambda_{\mathrm{em}}\right)$, accounts for light inelastically scattered from all relevant excitation wavelengths $\left(\lambda_{\text {ex }}\right)$ into an emission wavelength, $\lambda_{\mathrm{em}}$ :

$$
b^{R}\left(\lambda_{\mathrm{em}}\right)=\int_{\lambda_{\mathrm{ex}}} a^{R}\left(\lambda_{\mathrm{ex}}\right) f^{R}\left(\lambda_{\mathrm{ex}} \rightarrow \lambda_{\mathrm{em}}\right) \mathrm{d} \lambda_{\mathrm{ex}} .
$$

Here, $f^{R}\left(\lambda_{\text {ex }} \rightarrow \lambda_{\text {em }}\right)$ is the wavelength redistribution function, which is normalized to unity over all emission bands and whose shape was first described by Walrafen [7] for pure seawater. We follow the convention of Mobley [34] and define the "Raman absorption coefficient," $a^{R}\left(\lambda_{\text {ex }}\right)$, which quantifies the loss of radiant energy at the excitation wavelength due to inelastic Raman scatter. Bartlett et al. [10] provided the most recent measurements of the Raman absorption coefficient in seawater:

$$
a^{R}\left(\lambda_{\mathrm{ex}}\right)=2.7 \times 10^{-4}\left(\frac{\lambda_{\mathrm{ex}}}{488}\right)^{-5.3} .
$$

Finally, the Raman scattered radiance in the upward direction $\left(L_{u, R}\right)$ emanating from a subsurface layer between $z_{1}$ and $z_{1}+\Delta z$ at the emission band $\lambda_{\mathrm{em}}$ is

$$
\begin{aligned}
& L_{u, R}\left(z_{1}, \Delta z, \lambda_{\mathrm{em}}\right) \\
& \quad=\tilde{\beta}^{R}\left(\theta_{s} \rightarrow \pi\right) \int_{z_{1}+\Delta z}^{z_{1}} \int_{\lambda_{\mathrm{ex}}} b^{R}\left(\lambda_{\mathrm{em}}\right) E_{d}\left(z, \lambda_{\mathrm{ex}}\right) e^{-K_{d} z} \mathrm{~d} \lambda_{\mathrm{ex}} \mathrm{d} z,
\end{aligned}
$$

where $\tilde{\beta}^{r}\left(\theta_{s} \rightarrow \pi\right)$ is the Raman phase function, $E_{d}\left(z, \lambda_{\text {ex }}\right)$ is the downwelling irradiance at the relevant excitation wavelengths, and $K_{d}$ is the attenuation coefficient of downwelling irradiance. Integrating over all depths and assuming vertically homogeneous optical properties and single scattering, the Raman component of upwelling radiance just beneath the sea surface equals

$$
L_{u, R}\left(0^{-}, \lambda_{\mathrm{em}}\right)=\frac{\tilde{\beta}^{r}\left(\theta_{s} \rightarrow \pi\right) b_{r}\left(\lambda_{\mathrm{em}}\right) E_{d}\left(0^{+}, \lambda_{\mathrm{ex}}\right)}{\left(K_{d}\left(\lambda_{\mathrm{ex}}\right)+\kappa_{L}\left(\lambda_{\mathrm{em}}\right)\right)} .
$$

In this equation, $\kappa_{L}\left(\lambda_{\text {em }}\right)$ is the attenuation coefficient for upwelling radiance at emission wavelength $\lambda_{\mathrm{em}}$, and both the $E_{d}$ and $K_{d}$ are averaged over all excitation bands (width less than $20 \mathrm{~nm}$ for visible wavelengths $<600 \mathrm{~nm}$ ). For satellite applications, integration of $L_{u, R}\left(0^{-}, \lambda_{\mathrm{em}}\right)$ over the width of each satellite band is required ( $10 \mathrm{~nm}$ for MODIS), which is approximated by substituting band-averaged $b_{r}$ and $\kappa_{L}$ in Eq. (6). Similar expressions for the Raman contribution to upwelled radiance have been derived in previous studies $[\underline{10}, \underline{18}, \underline{21}, \underline{35}, \underline{36}$.

Transmitting the radiance across the air-sea interface and normalizing to incident downwelling irradiance, $E_{d}\left(0^{+}, \lambda_{\mathrm{em}}\right)$, yields an equivalent remote sensing reflectance:

$$
\begin{aligned}
& R_{\mathrm{rs}, \operatorname{Raman}}\left(0^{+}, \lambda_{\mathrm{em}}\right) \\
& =\frac{t^{2}}{n^{2}} \frac{\tilde{\beta}^{r}\left(\theta_{s} \rightarrow \pi\right) b_{r}\left(\lambda_{\mathrm{em}}\right) E_{d}\left(0^{+}, \lambda_{\mathrm{ex}}\right)}{\left(K_{d}\left(\lambda_{\mathrm{ex}}\right)+\kappa_{L}\left(\lambda_{\mathrm{em}}\right)\right) E_{d}\left(0^{+}, \lambda_{\mathrm{em}}\right)} \\
& \quad \times\left[1+\frac{b_{b}\left(\lambda_{\mathrm{ex}}\right)}{\mu_{u}\left(K_{d}\left(\lambda_{\mathrm{ex}}\right)+\kappa\left(\lambda_{\mathrm{ex}}\right)\right)}+\frac{b_{b}\left(\lambda_{\mathrm{em}}\right)}{2 \mu_{u} \kappa\left(\lambda_{\mathrm{em}}\right)}\right] .
\end{aligned}
$$

The additional terms in brackets on the right-hand side of Eq. (7) account for higher orders of scattering (e.g., Raman scattered light in the downward direction, then elastically backscattered into the upwelling stream) and are derived in detail elsewhere $[18,22]$. 
In practice, the approach presented in Eq. (7) for estimating the Raman component of $R_{\mathrm{rs}}(\lambda)$ requires estimates of IOPs $\left(a\right.$ and $\left.b_{b}\right)$ and diffuse attenuation ( $K$-functions). Therefore, an initial semianalytical inversion must be made to provide an estimate of the total absorption and backscattering coefficients. Attenuation coefficients are then calculated as

$$
K_{d}(\lambda)=\frac{a(\lambda)+b_{b}(\lambda)}{\mu_{d}} \quad \text { and } \quad \kappa(\lambda)=\frac{a(\lambda)+b_{b}(\lambda)}{\mu_{u}},
$$

$\mu_{d}$ and $\mu_{u}$ are the mean cosine of downwelling and upwelling light, respectively, and the latter is set as 0.5 as the light field is assumed similar to that of isotropic light [22], and the mean cosine for downwelling irradiance is calculated following Gordon [37] and Lee et al. [38].

The semianalytical inversion models used here require specification of eigenfunctions describing the spectral shape of IOPs. Therefore, another consideration that arises is extension of the eigenfunctions to the UV region where UV irradiance excites Raman emission in the visible. CDOM absorption and particulate backscattering can be extrapolated to the UV with simple exponential or power laws that adequately capture their spectral behavior (e.g., [27]). Absorption by phytoplankton in the UV is more complicated and variable, as UV absorbing substances of phytoplankton (e.g., mycosporine-like amino acids) can vary independently of other pigments (e.g., $[39,40])$. Here, we assume that $a_{p h}$ for $\lambda<$ $412 \mathrm{~nm}$ is spectrally flat, which may reflect a "mean" spectrum (see [40]).

\section{Results}

\section{A. Results from Forward Simulations}

Output from radiative transfer calculations provide a complete and exact IOP/AOP dataset to develop and test the approach for estimating the Raman component of $R_{\mathrm{rs}}\left(\lambda_{\mathrm{em}}\right)$ outlined in the previous section. Figure 1(a) shows simulated reflectance spectra over a wide range of $C h l$ with and without Raman scattering included (red dotted and black solid lines, respectively). The contribution from Raman scattered light can be assessed as the ratio of $R_{\mathrm{rs}, R}(\lambda) / R_{\mathrm{rs}}(\lambda)$, which increases with increasing wavelength, and with decreasing pigment concentration [Fig. 1(b)]. Specifically, the presence of Raman scatter enhances $R_{\mathrm{rs}}$ by $10 \%$ or less for $\lambda<500 \mathrm{~nm}$, while at longer wavelengths Raman scattered light can account for nearly $25 \%$ of the total $R_{\mathrm{rs}}$ in the clearest waters [Fig. 1(b)]. Importantly, $R_{\mathrm{rs}, R}(\lambda)$ is not spectrally flat, particularly in the blue to green spectral region. The magnitudes of the Raman contribution to reflectance shown here are consistent with previous studies (e.g., [12]) and are also a weak function of solar geometry and cloudiness.

\section{B. Inversion Using Semianalytical Models}

The simulated $R_{\mathrm{rs}}(\lambda)$ can be used as input to semianalytical inversion models for estimation of IOPs in the presence or absence of Raman scatter. In this work, we have chosen to use the GSM [30] and QAA [31] models, two approaches currently used by NA $\overline{S A}$ to produce satellite evaluation products.

These models return various component absorption and backscattering properties. QAA provides estimates of phytoplankton absorption at $443 \mathrm{~nm}$, $a_{p h}(443)$, while GSM provides an estimate of $C h l$. Both models return absorption by colored dissolved and detrital matter at $443 \mathrm{~nm}, a_{\mathrm{CDM}}(443)$, and the particulate backscattering coefficient at $443 \mathrm{~nm}$, $b_{b p}(443)$. Inversion results using the simulated reflectances are shown in Fig. 2. Biases exist in all retrieved parameters due to differing bio-optical relationships specified in the forward simulations (HydroLight) versus those assumed in the GSM and QAA inversion models. These differences could be easily reconciled by using the same expressions
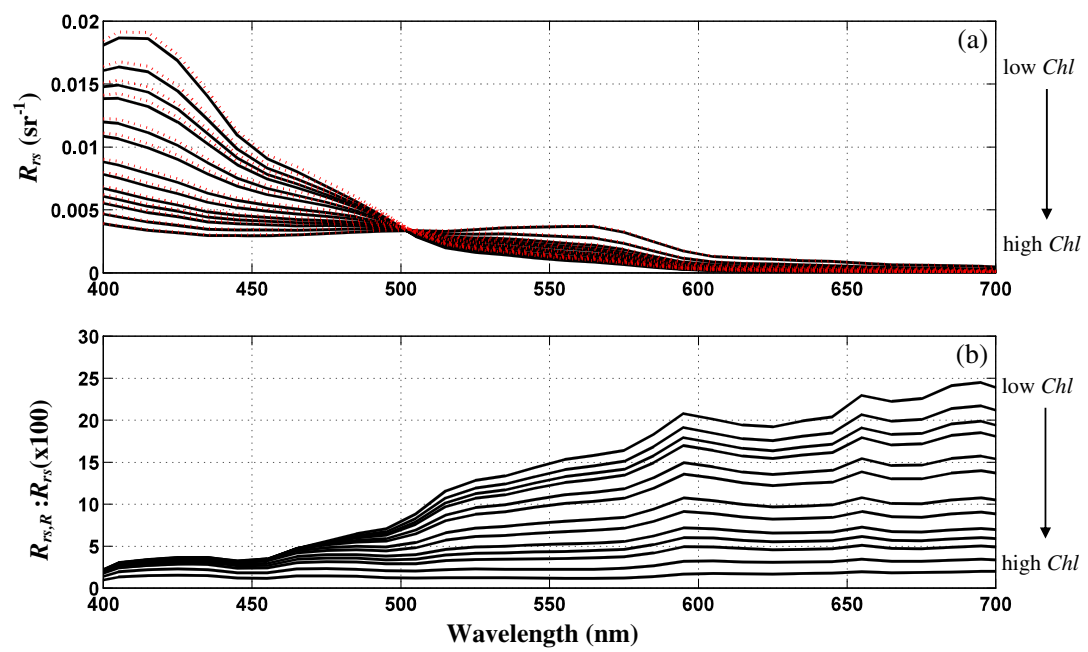

Fig. 1. Spectral remote sensing reflectance from HydroLight simulations. (a) $R_{\mathrm{rs}}(\lambda)$ for varying Chl for cases which include Raman scatter (dotted red lines) and which do not include Raman scatter (solid black lines). (b) Percent contribution of Raman scatter to $R_{\mathrm{rs}}(\lambda)$ expressed as the ratio of $R_{\mathrm{rs}, R}(\lambda): R_{\mathrm{rs}}(\lambda)$ times 100 . Details of simulations are described in Section $\underline{2}$. 

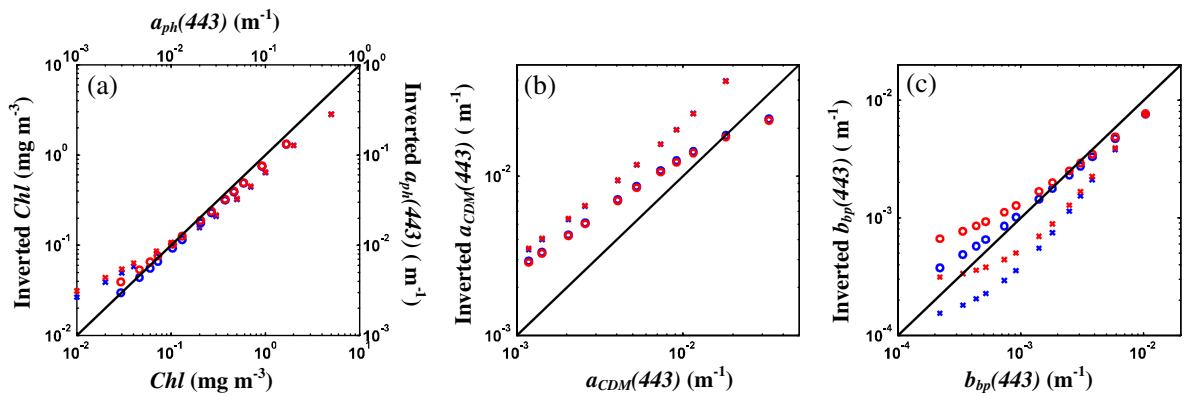

Fig. 2. IOPs from inversion of HydroLight $R_{\mathrm{rs}}(\lambda)$ with and without Raman scatter included. Values plotted on the abscissae in each panel are taken from HydroLight and considered the "true" IOP value. (a) Chl from GSM (bottom and left axes) and $a_{p h}(443)$ from QAA (top and right axes); (b) $a_{\mathrm{CDM}}(443)$; (c) $b_{b p}(443)$. In each panel, " $x$ " and "o" represent GSM and QAA retrievals, respectively. Red and blue symbols represent inversions of $R_{\mathrm{rs}}(\lambda)$ with and without Raman scatter included, respectively.

for phytoplankton, dissolved and detrital absorption, and particulate backscattering in the forward and inverse models (not shown). However, it is the relative difference in retrieved IOPs due to Raman scatter that we focus on in this work. The relative error (or bias) is defined as $\left(\mathrm{IOP}_{\text {uncorr }}-\mathrm{IOP}_{\text {corr }}\right) / \mathrm{IOP}_{\text {corr }} * 100$, where IOP is any retrieved IOP and the subscripts uncorr and corr refer to IOPs estimated from reflectance spectra that are uncorrected or corrected for Raman, respectively. As a control, simulated spectra with and without Raman scattering explicitly included are substituted in place of uncorrected and corrected reflectances, respectively. Results from these control runs (black lines in each panel of Fig. 3 shows that (1) the relative error in each IOP due to Raman scattering differs greatly between each IOP, (2) errors differ between inversion models (GSM versus
QAA), (3) errors are greatest at low $C h l$ and decrease with increasing $C h l$, and (4) errors are greatest in the retrieval of $b_{b p}$ (443) [Figs. 3(a)-3(f)]. Chl and $a_{p h}$ (443) are overestimated by $\sim 1 \overline{5 \%}-\overline{25 \%}$ under the most oligotrophic conditions $\left(C h l<0.02 \mathrm{mg} \mathrm{m}^{-3}\right)$, and decrease to $\sim 5 \%$ when $C h l>0.3 \mathrm{mg} \mathrm{m}^{-3}$ [Figs. 3(a) and $3(\mathrm{~d})$ ]. Errors in $a_{\mathrm{CDM}}(443)$ are negligible across all trophic conditions [Figs. 3(b) and 3(e)]. Errors in $b_{b p}$ (443), however, can be $>\overline{100 \%}$ under ultraoligotrophic conditions and are still $\sim 20 \%$ when $C h l>$ $0.3 \mathrm{mg} \mathrm{m}^{-3}$ [Fig. $\underline{3(\mathrm{c})}$ ].

\section{Evaluation of Raman Contribution Removal Scheme}

The steps outlined in Section 2.C [Eq. (7)] result in an estimate of $R_{\mathrm{rs}, R}(\lambda)$. We can provide a measure of validation for the approach using the simulated dataset by comparing estimates of $R_{\mathrm{rs}, R}(\lambda)$ with the absolute
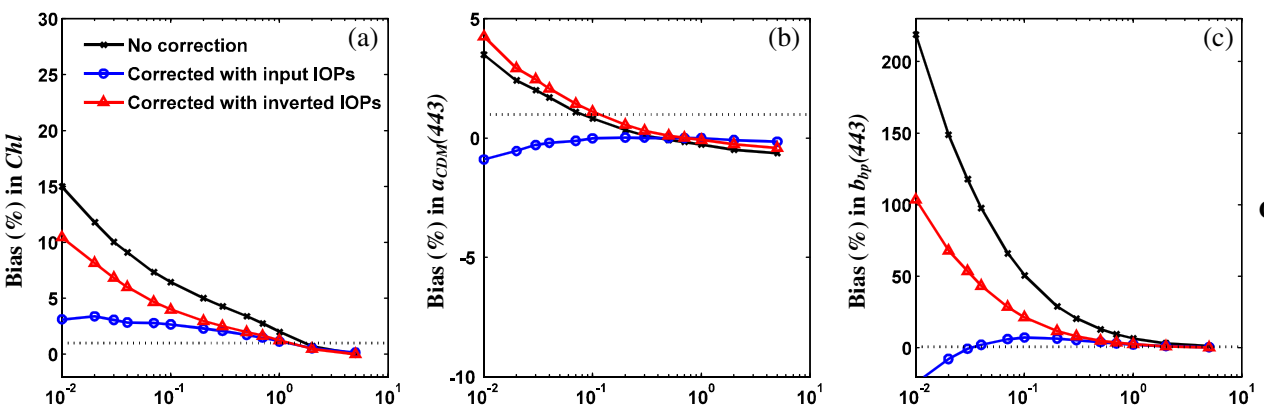

GSM
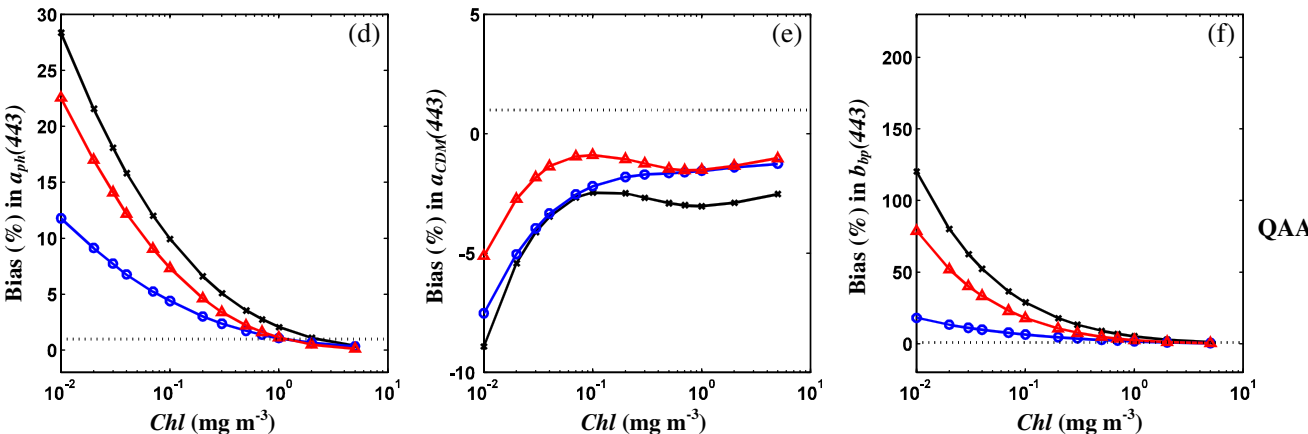

Fig. 3. Relative error in inverted IOPs due to Raman scatter as a function of chlorophyll concentration. Bias is calculated as normalized difference (\%) between each retrieved IOP from $R_{\mathrm{rs}}(\lambda)$ with and without Raman scatter included. In each panel three curves are shown that represent: error in retrievals using uncorrected Rrs (black line), error in retrievals after correction of Rrs with exact IOPs (blue line), and error in retrievals after correction of Rrs with estimated IOPs from either GSM or QAA (red lines) IOPs in the top and bottom row, respectively. (a) GSM Chl; (b) GSM $a_{\mathrm{CDM}}(443)$; (c) GSM $b_{b p}(443)$; (d) QAA $a_{p h}$ (443); e, QAA $a_{\mathrm{CDM}}(443)$; and (f) QAA $b_{b p}(443)$. 


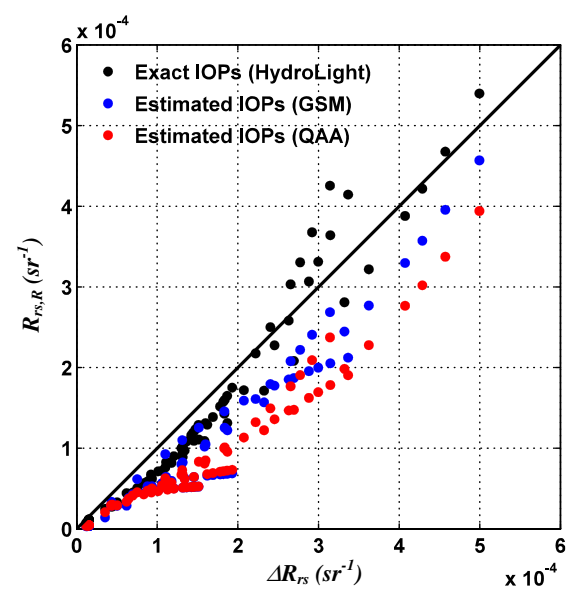

Fig. 4. Estimation of Raman component of $R_{\mathrm{rs}}(\lambda) . R_{\mathrm{rs}, R}$ is directly estimated from Eq. (7). $\Delta R_{\mathrm{rs}}$ is the arithmetic difference between radiative transfer simulations with and without Raman scattering included. Results for all visible satellite wave bands are shown together. Diagonal line is 1:1 line.

difference in $R_{\mathrm{rs}}(\lambda)$ taken from consecutive runs with and without Raman scattering included $\left(\Delta R_{\mathrm{rs}}\right)$ (Fig. 4). In the best case scenario, exact IOP inputs from HydroLight can be used to estimate $R_{\mathrm{rs}, R}(\lambda)$ and the resultant agreement with $\Delta R_{\mathrm{rs}}$ is very good across the whole range of anticipated $R_{\mathrm{rs}}$ and over all visible satellite wave bands $r^{2}=0.95$, slope $=1.08$, mean bias $=19 \%)$. When $R_{\mathrm{rs}, R}(\lambda)$ is estimated using inverted IOPs rather than those taken directly from HydroLight, predictability is slightly degraded with differences depending upon which inversion model is
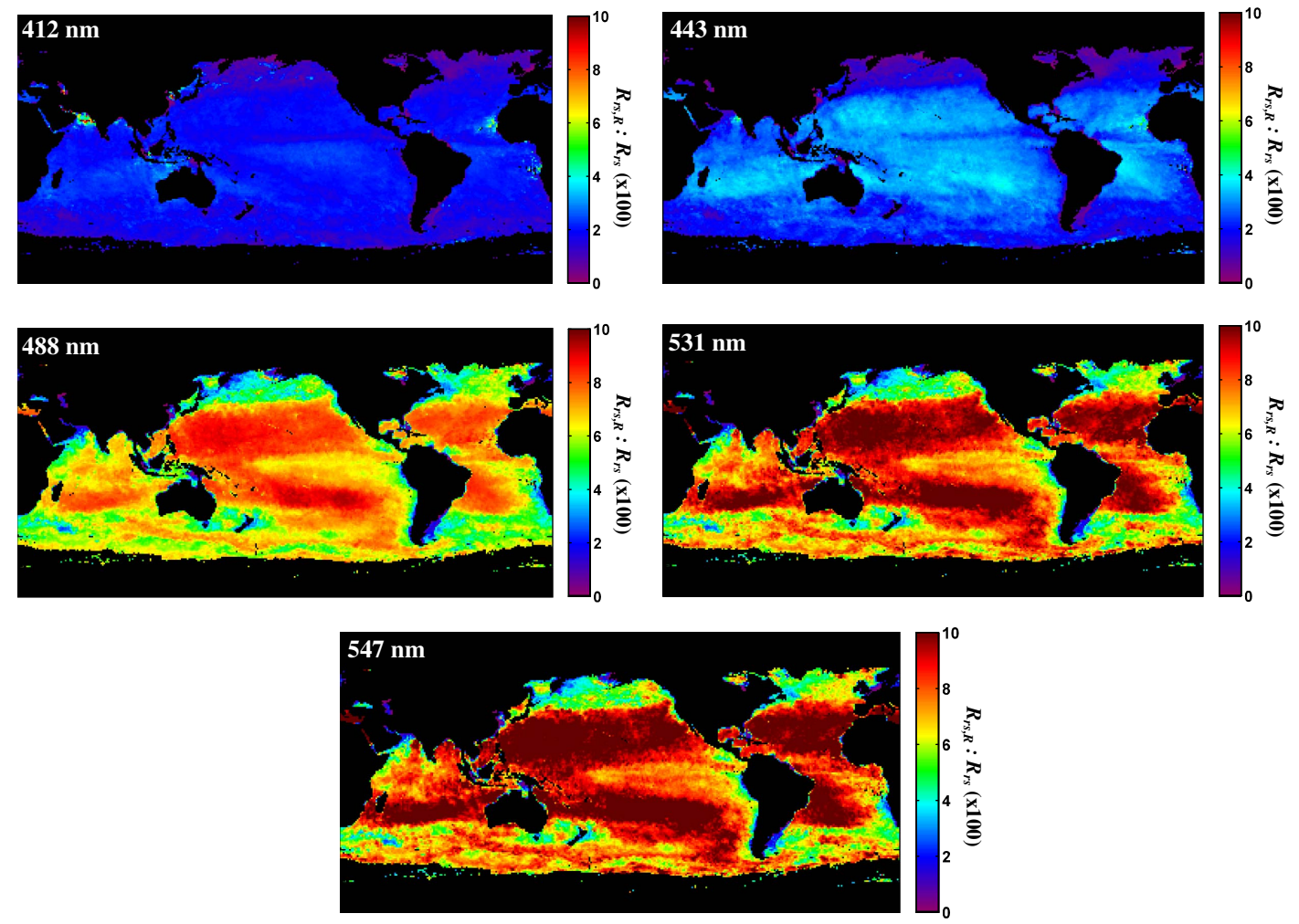

Fig. 5. Fractional contribution of Raman component to total $R_{\mathrm{rs}}(\lambda)$ calculated for a single L3 monthly MODIS composite image (October 2004). Values are expressed as a percentage (\%) and each panel shows different MODIS wave bands in the visible. 


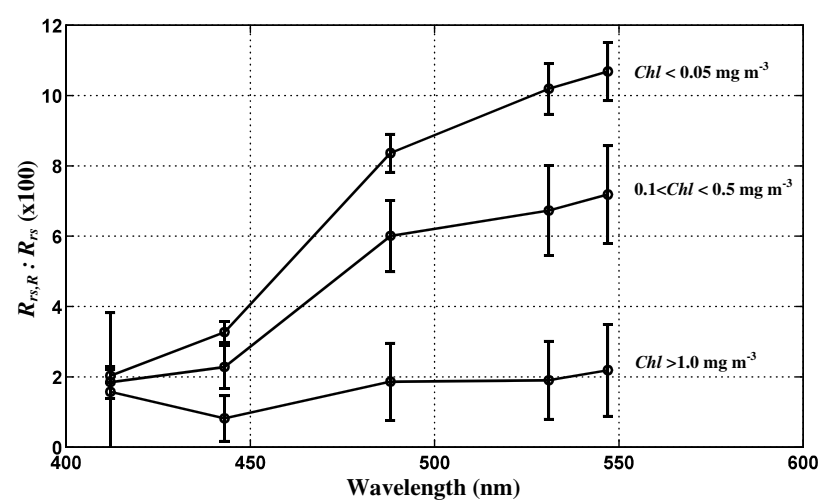

Fig. 6. Fractional contribution of Raman scattered radiance to total $R_{\mathrm{rs}}(\lambda)$ for various ranges of observed satellite $C h l$ (October 2004). Chl bins are for values $<0.05 \mathrm{mg} \mathrm{m}^{-3}$ (top curve), $0.1<$ $C h l<0.5 \mathrm{mg} \mathrm{m}^{-3}$ (middle curve), and $C h l>1.0 \mathrm{mg} \mathrm{m}^{-3}$ (bottom curve). Error bars represent ranges of variability within each $C h l$ bin. Results for MODIS wave bands $>551 \mathrm{~nm}$ not shown, due to contamination by $C h l$ fluorescence.

ocean visible wave bands, except in the red (667 and $678 \mathrm{~nm}$ ), where the contribution from Chl fluorescence can be significant. Three general observations are evident and are consistent with results from the simulated data: (1) values range from $<1 \%$ to $10 \%$, (2) contributions from Raman increase at longer wavelengths, and (3) contributions from Raman tend to be a decreasing function of biomass. The latter point can be seen as higher relative contribution to $R_{\mathrm{rs}}(\lambda)$ from Raman scatter in the oligotrophic gyres and lower values in productive high latitude waters and areas of strong upwelling (Fig. 5). Further, if the global data are binned into three broad $C h l$ ranges, which can loosely be categorized as oligotrophic, mesotrophic, and eutrophic $\left(C h l<0.05 \mathrm{mg} \mathrm{m}^{-3}\right.$, $0.1<C h l<0.5 \mathrm{mg} \mathrm{m}^{-3}$, and $C h l>1.0 \mathrm{mg} \mathrm{m}^{-3}$, respectively), we can collapse the spatial information and look at results across all wave bands (Fig. $\underline{6}$ ). The spectral variability is similar to that seen in the simulated data presented in Fig. 1, where the Raman component contributes very little $(\sim 2 \%)$ to $R_{\mathrm{rs}}$ at $412 \mathrm{~nm}$, but then increases more rapidly for clearer water. Maximal $R_{\mathrm{rs}, R} / R_{\mathrm{rs}}$ is observed at $547 \mathrm{~nm}$ (formerly referred to as $551 \mathrm{~nm}$ ) among the bands analyzed here and is approximately $11 \%, 7 \%$, and $2 \%$ for oligotrophic, mesotrophic, and eutrophic waters.

Last, we remove the estimated $R_{\mathrm{rs}, R}(\lambda)$ from satellite $R_{\mathrm{rs}}(\lambda)$ and re-invert the global fields to provide Raman-corrected IOP estimates (Fig. 7). The global distribution of IOPs before and after correction show varied responses. For the GSM model, median $C h l$ decreases only slightly $(\sim 8 \%)$ from $0.12 \mathrm{mg} \mathrm{m}^{-3}$ to $0.11 \mathrm{mg} \mathrm{m}^{-3}$ after correction for Raman [Fig. 7(a)]. Median phytoplankton absorption $\left(a_{p h}(443)\right)$ estimated from the QAA decreases similarly (8\%) following correction [Fig. 7(d)]. Retrievals of CDOM and detrital absorption, $\overline{a_{\mathrm{CDM}}}(443)$, are particularly insensitive to the presence of Raman scattering and only change by $<3 \%$ for either inversion model [Figs. 7(b) and 7(e)]. The largest differences resulting from the Raman correction are observed in $b_{b p}(443)$, similar to that seen in the simulated dataset. Global distributions of $b_{b p}(443)$ from GSM and QAA, precorrection and postcorrection are shown in Figs. 7(c) and $7(\mathrm{f})$. The distributions for each model are shifted downward after Raman correction, and the overall distributions become flatter. The changes in $b_{b p}(443)$ are further examined in Fig. $\underline{8}$, which shows
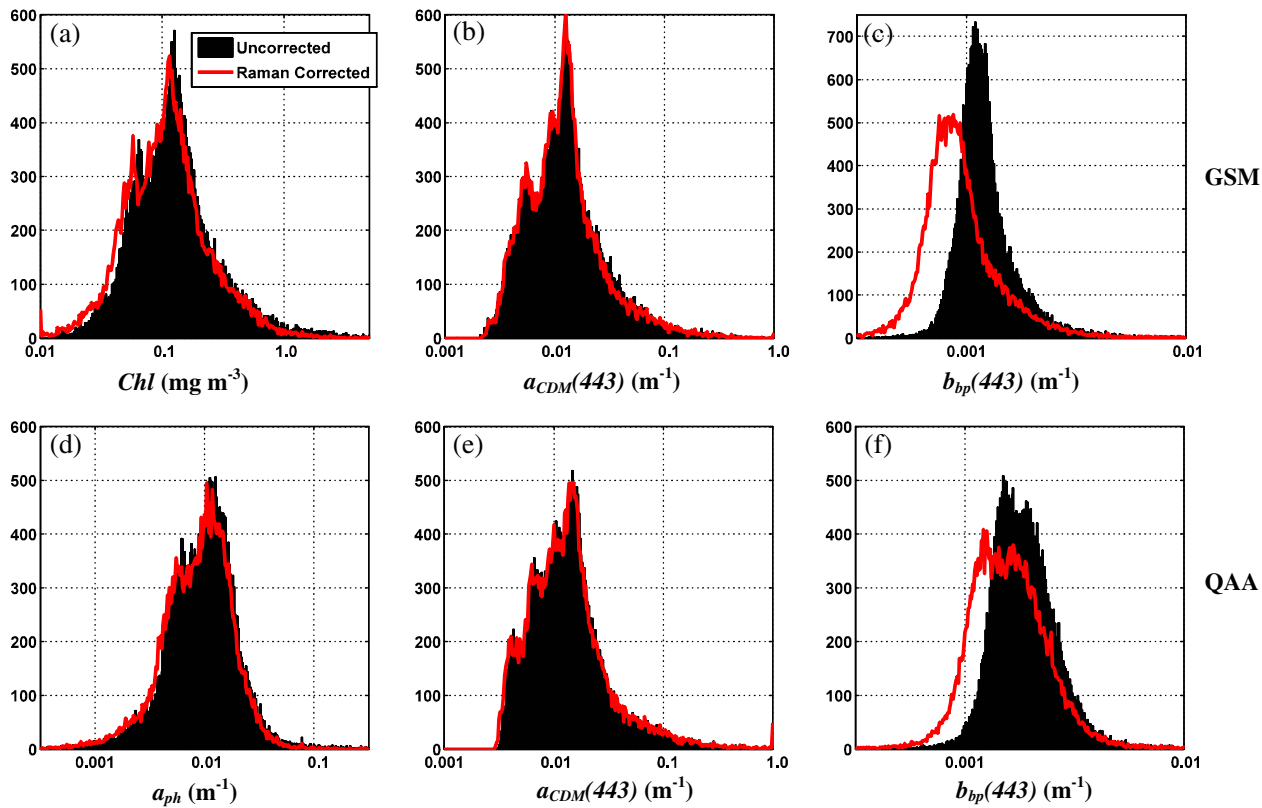

Fig. 7. Histograms of global IOP retrievals for a single L3 monthly composite (October 2004). Top panels show GSM retrievals of (a) Chl; (b) $a_{\mathrm{CDM}}(443)$; (c) $b_{b p}$ (443). Bottom panels show QAA retrievals for (d) $a_{p h}(443)$; (e) $a_{\mathrm{CDM}}(443)$; (f) $b_{b p}$ (443). In each panel, the black histogram is from monthly values estimated without any correction for Raman scattering (the default), and the red line is from inversion after removing the Raman contribution to $R_{\mathrm{rs}}(\lambda)$. 

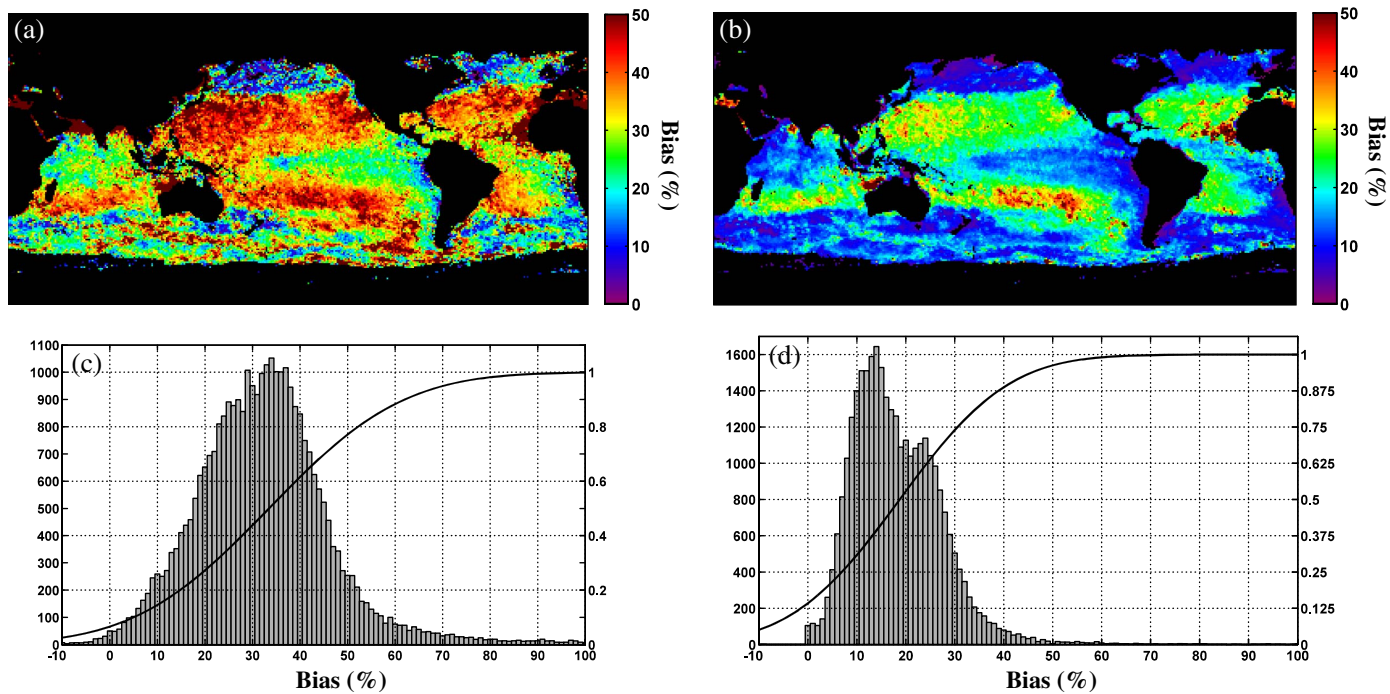

Fig. 8. Comparison of satellite $b_{b p}(443)$ inversions before and after removal of Raman component of remote sensing reflectance, $R_{\mathrm{rs} . R}(\lambda)$. (a) and (b) show the spatial distribution of error in $b_{b p}(443)$ due to Raman for the GSM and QAA inversions, respectively. (c) and (d) are histograms of each respective image. Black lines are cumulative distribution functions of each field. Bias is calculated as normalized difference between $b_{b p}(443)$ estimated from satellite $R_{\mathrm{rs}}(\lambda)$ with and without Raman scatter included ( $\times 100$ to express as a percentage).

the overall distribution of the difference due to Raman correction (expressed as a relative bias, \%), as well as the spatial distribution of this bias. Differences are consistently higher for GSM $b_{b p}(443)$ retrievals [Fig. 8(a)] than for the QAA. Raman correction results in values that are much lower across most of the mid-latitudes, and to a lesser extent at high latitudes. The median bias is $\sim 30 \%$ and $20 \%$ for the GSM and QAA, respectively, and suggests that $b_{b p}(443)$ is significantly overestimated over much of the ocean when using either model. While these "average" biases may not seem too large on their own, it is important to note that up to $30 \%$ of the ocean has errors due to Raman in excess of $50 \%$ [see CDF in Fig. 8(c)].

\section{Discussion and Conclusions}

Failure to account for Raman scattering can lead to large errors in some semianalytically inverted properties (e.g., particulate backscattering). Here, we have developed a straightforward approximation for the contribution of Raman scattering to remote sensing reflectance in satellite ocean color wave bands (e.g., MODIS). This method can be applied in conjunction with inversion of ocean color reflectance data to yield more accurate IOPs which are related only to the elastically backscattered component of $R_{\mathrm{rs}}(\lambda)$. In addition to the observed $R_{\mathrm{rs}}(\lambda)$, the only external inputs that are required are spectral irradiance in the Raman excitation and emission bands, $E_{d}\left(\lambda_{\text {ex }}\right)$ and $E_{d}\left(\lambda_{\text {em }}\right)$. These values can be obtained directly from a solar irradiance model (e.g., [41]) with knowledge of atmospheric properties, such as that carried out for operational ocean color processing. As an example in the work presented here, we have used combined irradiance data in the UV region from the OMI sensor with spectrally decomposed irradiances in the visible which are derived from the standard MODIS iPAR product.

The approach presented here is not without its limitations, and significant biases due to Raman remain in inverted quantities even after correction. However, nearly all of the error due to Raman scatter can be corrected for, and removed if all inherent and apparent optical property input data is known accurately, such as the example shown with simulated data (blue lines in Fig. 3). This suggests that inability to completely correct for the Raman scattering is not due to the method presented in Eq. (7), but rather to the inversion schemes themselves. Conceptually, this procedure should be run iteratively, with each iteration removing slightly more Raman-associated bias. What we found in doing so, however, is that the exercise converged after a single iteration. Inspection of Eqs. (7) and (8) show that the IOPs required to estimate $R_{\mathrm{rs}, R}(\lambda)$ are total absorption, $a(\lambda)$, and backscattering, $b_{b}(\lambda)$. Since $a(\lambda) \gg b_{b}(\lambda)$ in the oligotrophic ocean, $R_{\mathrm{rs}, R}(\lambda)$ is primarily dependent upon total absorption, which is retrieved relatively well, particularly when including pure seawater absorption. So, while errors in $b_{b p}(\lambda)$ may remain relatively large, the correction cannot improve them with subsequent iterations. Similar findings were reported by Loisel and Stramski [23]. Interestingly, inversion estimates of $a_{\mathrm{CDM}}(443)$ are relatively insensitive to the presence of Raman and its correction. This is most likely because $a_{\mathrm{CDM}}(\lambda)$ is weighted toward short wavelengths (UV and blue/violet) and there is very little inelastic contribution to $R_{\mathrm{rs}}(\lambda)$ in this region (Fig. 1). This, in turn, results from the strongly attenuated excitation irradiances for these emission bands at the sea surface (peak excitation wavelengths for the 412 and $443 \mathrm{~nm}$ MODIS bands are at $\sim 365$ and $387 \mathrm{~nm}$, respectively). 
There are other confounding factors for unraveling the effect of Raman scattering on ocean color inversion models. For example, field data that is used to parameterize models (e.g., NASA's NOMAD, [42]) already contain inelastic scattering contributions from Raman. The process of optimizing the inversion models $[27,31]$ should mitigate some of the Raman effect, such that errors estimated here may be viewed as upper bounds. Further, if inversion model coefficients have been optimized to match $R_{\mathrm{rs}}$ containing Raman with coincident IOPs, then subsequent removal of the Raman component will necessarily degrade the model's ability to correctly retrieve the IOPs. In this context, a more appropriate test for Raman effects on inversion models would be to evaluate the retrieval of IOPs from corrected $R_{\mathrm{rs}}$ with a model that has been "re"-optimized with a dataset that has had Raman contributions to $R_{\mathrm{rs}}$ removed.

Alternative approaches that employ varying degrees of complexity exist to correct $R_{\mathrm{rs}}(\lambda)$ for Raman scattering (e.g., $[22,23,43,44])$. For inversion models, such as the GSM, which rely on a minimization scheme, the Raman terms could simply be included explicitly within the inversion. Doing so may provide a more compact, "eloquent" approach, but there is no reason to expect any different results from those presented here. For models such as the QAA, this would not be possible without significant reworking of the model. A more common approach applicable to any inversion scheme and which has been applied in previous studies $[23,43,44]$ draws directly from radiative transfer simulations. If a representative suite of simulations with and without Raman scatter is generated, it is possible to construct a lookup table or to parameterize $R_{\mathrm{rs} R}(\lambda)$ as a function of environmental (e.g., $C h l$ ) or optical parameters (e.g., $R_{\mathrm{rs}}$ ). This approach can work well, but is subject to limitations inherent in the radiative transfer model used to generate the simulations (e.g., Case I-type assumptions). Further, it is not straightforward to evaluate how well this kind of approach works because simulated data are again required to do so. In contrast, the approach presented here is free of these constraints, but the trade-off is that the correction is limited by inaccurate IOP retrievals and their extension to the excitation wavelengths as required to estimate $R_{\mathrm{rs}, R}(\lambda)$ via Eq. (7). Empirical "bandratio" type algorithms for $C h l[\overline{4} 5]$ or diffuse attenuation $[38,46]$, which are not examined here, may also suffer from biases due to Raman scatter.

The global patterns and conclusions drawn here result from the analysis of an illustrative example of a single monthly satellite composite. This approach must be applied to a longer time period in order to better characterize the extent of bias attributed to Raman scatter in the global ocean. The steep dependence of the Raman bias on Chl dictates how important it may be for a particular application. For example, regional studies in highly productive areas might ignore Raman effects with only modest errors in retrieved IOPs. In contrast, targeted studies of the oligotrophic gyres would be well-served to consider Raman contributions to $R_{\mathrm{rs}}(\lambda)$ and inverted IOPs. The results conveyed in Figs. $\underline{7}$ and $\underline{8}$ provide an illustrative example of global patterns. Last, the biases calculated must be propagated through to the biogeochemical quantities of interest to evaluate the significance of this process. For example, relationships that estimate particle stocks from $b_{b p}$ (e.g., $[47, \underline{48}]$ ) will be affected in direct proportion to the Raman bias. However, some applications [e.g., calculation of net primary production (NPP)] will require more detailed sensitivity analyses to understand the impact of the Raman bias.

Funding for this work was provided by NASA grant NNX12AJ13G to EB and TKW. The authors wish to thank Curtis Mobley for many helpful discussions in early stages of this work and comments on a previous version of the manuscript. Two anonymous reviewers also greatly helped to improve the manuscript. Thanks also to Emmanuel Devred for sharing Fortran code.

\section{References}

1. H. R. Gordon, O. B. Brown, R. H. Evans, J. W. Brown, R. C. Smith, K. S. Baker, and D. K. Clark, "A semianalytic radiance model of ocean color,” J. Geophys. Res. 93, 10909-10924 (1988).

2. C. R. McClain, S. R. Signorini, and J. R. Christian, "Subtropical gyre variability observed by ocean-color satellites,” Deep-Sea Research, Part II 51, 281-301 (2004).

3. D. G. Boyce, M. R. Lewis, and B. Worm, "Global phytoplankton decline over the past century," Nature 466, 591-596 (2010).

4. M. J. Behrenfeld, R. T. O’Malley, D. A. Siegel, C. R. McClain, J. L. Sarmiento, G. C. Feldman, A. J. Milligan, P. G. Falkowski, R. M. Letelier, and E. S. Boss, "Climate-driven trends in contemporary ocean productivity," Nature 444, 752-755 (2006).

5. C. V. Raman, "On the molecular scattering of light in water and the colour of the sea," Proc. R. Soc. London Ser. A 101, 64-80 (1922).

6. C. V. Raman and K. S. Krishnan, "A new type of secondary radiation," Nature 121, 501-502 (1928).

7. G. E. Walrafen, "Raman spectral studies of effects of temperature on water structure," J. Chem. Phys. 47, 114-126 (1967).

8. S. Sugihara, M. Kishino, and N. Okami, "Contribution of Raman scattering to upward irradiance in the sea," J. Oceanogr. Soc. Jpn. 40, 397-404 (1984).

9. B. R. Marshall and R. C. Smith, "Raman-scattering and inwater ocean optical-properties," Appl. Opt. 29, 71-84 (1990).

10. J. S. Bartlett, K. J. Voss, S. Sathyendranath, and A. Vodacek, "Raman scattering by pure water and seawater," Appl. Opt. 37, 3324-3332 (1998).

11. R. A. Desiderio, "Application of the Raman scattering coefficient of water to calculations in marine optics," Appl. Opt. 39, 1893-1894 (2000).

12. H. R. Gordon, "Contribution of Raman scattering to water-leaving radiance: a reexamination," Appl. Opt. 38, 3166-3174 (1999).

13. R. H. Stavn and A. D. Weidemann, "Optical modeling of clear ocean light fields-Raman scattering effects," Appl. Opt. 27, 4002-4011 (1988).

14. Y. T. Ge, H. R. Gordon, and K. J. Voss, "Simulation of inelasticscattering contributions to the irradiance field in the ocean-variation in Fraunhofer line depths," Appl. Opt. 32, 4028-4036 (1993).

15. K. J. Waters, "Effects of Raman-scattering on the waterleaving radiance," J. Geophys. Res. 100, 13151-13161 (1995).

16. Y. T. Ge, K. J. Voss, and H. R. Gordon, "In-situ measurements of inelastic light-scattering in Monterey Bay using solar Fraunhofer lines,” J. Geophys. Res. 100, 13227-13236 (1995). 
17. C. M. Hu and K. J. Voss, "In situ measurements of Raman scattering in clear ocean water," Appl. Opt. 36, 6962-6967 (1997).

18. J. S. Bartlett, "The influence of Raman scattering by seawater and fluorescence by phytoplankton on ocean colour," M.S. thesis (Dalhousie University, 1996).

19. R. M. Pope and E. S. Fry, "Absorption spectrum (380-700 nm) of pure water. 2 . Integrating cavity measurements," Appl. Opt. 36, 8710-8723 (1997).

20. H. R. Gordon, M. R. Lewis, S. D. McLean, M. S. Twardowski, S. A. Freeman, K. J. Voss, and G. C. Boynton, "Spectra of particulate backscattering in natural waters," Opt. Express 17, 16192-16208 (2009).

21. Z. P. Lee, K. L. Carder, S. K. Hawes, R. G. Steward, T. G. Peacock, and C. O. Davis, "Model for the interpretation of hyperspectral remote-sensing reflectance," Appl. Opt. 33, 5721-5732 (1994).

22. S. Sathyendranath and T. Platt, "Ocean-color model incorporating transspectral processes," Appl. Opt. 37, 2216-2227 (1998).

23. H. Loisel and D. Stramski, "Estimation of the inherent optical properties of natural waters from the irradiance attenuation coefficient and reflectance in the presence of Raman scattering," Appl. Opt. 39, 3001-3011 (2000).

24. IOCCG, "Remote sensing of inherent optical properties: fundamentals, tests of algorithms, and applications" (International Ocean Colour Coordination Group, 2006), Report Number 5.

25. C. D. Mobley, B. Gentili, H. R. Gordon, Z. H. Jin, G. W. Kattawar, A. Morel, P. Reinersman, K. Stamnes, and R. H. Stavn, "Comparison of numerical-models for computing underwater light fields," Appl. Opt. 32, 7484-7504 (1993).

26. H. R. Gordon and A. Morel, Remote Assessment of Ocean Color for Interpretation of Satellite Visible Imagery. A Review (Springer-Verlag, 1983).

27. A. Morel and S. Maritorena, "Bio-optical properties of oceanic waters: a reappraisal," J. Geophys. Res. 106, 7163-7180 (2001).

28. R. C. Smith and K. S. Baker, "Optical-properties of the clearest natural waters (200-800 nm),” Appl. Opt. 20, 177-184 (1981).

29. P. Ricchiazzi, S. R. Yang, C. Gautier, and D. Sowle, "SBDART: a research and teaching software tool for plane-parallell radiative transfer in the Earth's atmosphere," Bull. Am. Meteorol. Soc. 79, 2101-2114 (1998).

30. S. Maritorena, D. A. Siegel, and A. R. Peterson, "Optimization of a semianalytical ocean color model for global-scale applications," Appl. Opt. 41, 2705-2714 (2002).

31. Z. P. Lee, K. L. Carder, and R. A. Arnone, "Deriving inherent optical properties from water color: a multiband quasianalytical algorithm for optically deep waters," Appl. Opt. 41, 5755-5772 (2002).

32. D. Pozdiakov and H. Grassl, Color of Inland and Coastal Waters: A Methodology for its Interpretation (Springer-Praxis, 2003), p. 192.

33. H. R. Gordon, "Diffuse reflectance of the ocean: the theory of its augmentation by chlorophyll a fluorescence at $685 \mathrm{~nm}$," Appl. Opt. 18, 1161-1166 (1979).
34. C. D. Mobley, Light and Water: Radiative Transfer in Natural Waters (Academic, 1994).

35. V. I. Haltrin and G. W. Kattawar, "Self-consistent solutions to the equation of transfer with elastic and inelastic-scattering in ocean optics: 1. Model," Appl. Opt. 32, 5356-5367 (1993).

36. C. Mobley, "Interpretation of Raman scattering computations," HydroLight Technical Note, 10 (2012).

37. H. R. Gordon, "Can the Lambert-Beer law be applied to the diffuse attenuation coefficient of ocean water?," Limnol. Oceanogr. 34, 1389-1409 (1989).

38. Z. P. Lee, M. Darecki, K. L. Carder, C. O. Davis, D. Stramski, and W. J. Rhea, "Diffuse attenuation coefficient of downwelling irradiance: an evaluation of remote sensing methods," J. Geophys. Res. 110, C02017 (2005).

39. J. R. Morrison and N. B. Nelson, "Seasonal cycle of phytoplankton UV absorption at the Bermuda Atlantic Time-series Study (BATS) site," Limnol. Oceanogr. 49, 215-224 (2004).

40. A. Bricaud, M. Babin, H. Claustre, J. Ras, and F. Tieche, "Light absorption properties and absorption budget of Southeast Pacific waters," J. Geophys. Res. 115, C08009 (2010).

41. W. W. Gregg and K. L. Carder, "A simple spectral solar irradiance model for cloudless maritime atmospheres," Limnol. Oceanogr. 35, 1657-1675 (1990).

42. P. J. Werdell and S. W. Bailey, "An improved in-situ bio-optical data set for ocean color algorithm development and satellite data product validation," Remote Sens. Environ. 98, 122-140 (2005).

43. Z. P. Lee, S. L. Shang, C. M. Hu, M. Lewis, R. Arnone, Y. H. Li, and B. Lubac, "Time series of bio-optical properties in a subtropical gyre: Implications for the evaluation of interannual trends of biogeochemical properties," J. Geophys. Res. 115 C09012 (2010).

44. Z. P. Lee, C. M. Hu, S. L. Shang, K. Du, M. Lewis, and R. Arnone, "Penetration of UV-visible solar light in the global oceans: insights from ocean color remote sensing," J. Geophys. Res.-Oceans, in review.

45. J. E. O’Reilly, S. Maritorena, B. G. Mitchell, D. A. Siegel, K. L. Carder, S. A. Garver, M. Kahru, and C. McClain, "Ocean color chlorophyll algorithms for SeaWiFS," J. Geophys. Res. 103, 24937-24953 (1998).

46. A. Morel, Y. Huot, B. Gentili, P. J. Werdell, S. B. Hooker, and B. A. Franz, "Examining the consistency of products derived from various ocean color sensors in open ocean (Case 1) waters in the perspective of a multi-sensor approach," Remote Sens. Environ. 111, 69-88 (2007).

47. D. Stramski, R. A. Reynolds, M. Babin, S. Kaczmarek, M. R. Lewis, R. Rottgers, A. Sciandra, M. Stramska, M. S. Twardowski, B. A. Franz, and H. Claustre, "Relationships between the surface concentration of particulate organic carbon and optical properties in the eastern South Pacific and eastern Atlantic Oceans," Biogeosciences 5, 171-201 (2008).

48. T. Westberry, M. J. Behrenfeld, D. A. Siegel, and E. Boss, "Carbon-based primary productivity modeling with vertically resolved photoacclimation," Global Biogeochem Cycles 22, GB2024 (2008). 